\title{
Demonstration of high-rate laser communications from a fast airborne platform
}

\author{
Florian Moll, Joachim Horwath, Amita Shrestha, Martin Brechtelsbauer, Christian Fuchs, Luis Martin Navajas, \\ Alberto Lozano Souto and Dionisio Diaz Gonzale
}

\begin{abstract}
In this paper, we report on the demonstration of a high-rate free-space optical communication downlink from a fast airborne platform to a ground station. The flight platform used was a Panavia Tornado with a laser communication terminal installed in an attached avionic demonstrator pod. A transportable optical ground station equipped with a free-space receiver frontend was used as the receiver station. Downlink wavelength for communication and uplink wavelength for beacon laser were chosen to be compatible with the C-band DWDM grid. New opto-mechanical tracking systems were developed and applied on both sides for link acquisition and stabilization. The flight tests were carried out at the end of November 2013 near the Airbus Defence \& Space location in Manching, Germany. The campaign successfully demonstrated the maturity and readiness of laser communication for aircraft downlinks at a data rate of $\mathbf{1 . 2 5}$ Gbit/s. We outline the experiment design based on link budget assessments, the developed opto-mechanical terminal technology, and the results of the flight campaign. The experiment itself focused on the tracking performance of the airborne terminal and the ground station. Performance could be measured at aircraft speeds up to Mach 0.7 and video data from an onboard camera was transmitted. Tracking accuracies of up to $20 \mu \mathrm{rad} \mathrm{rms}$ for the airborne terminal and the ground station were achieved at instantaneous tracking errors below $60 \mu \mathrm{rad}$ and $40 \mu \mathrm{rad}$, respectively. The tracking link worked up to a horizontal distance of $79 \mathrm{~km}$ and data transmission was possible up to $50 \mathrm{~km}$.
\end{abstract}

Index Terms-free-space optical communications, aeronautical communication, laser terminal, transportable optical ground station, aircraft downlink, project DODfast.

\section{INTRODUCTION}

C URRENT and future airborne payloads such as high resolution cameras and radar systems need high channel capacities to transmit their data from air to ground in near real-time. Especially in reconnaissance and surveillance missions, it is important to downlink huge amounts of data within very short contact times to a ground station during a flyby maneuver. Present-day aeronautical data links employ conventional radio frequency (RF) technology. However, the $\mathrm{RF}$ spectrum is limited and heavily regulated. Licenses for high data rate communications are not easy to obtain and are usually very expensive. This problem can be solved by using frequencies in the optical domain, which offer a broad spectrum and are not under regulation by the International Telecommunication Union. Furthermore, airborne and ground

F. Moll, A. Shrestha, M. Brechtelsbauer and C. Fuchs are with the Institute of Communications and Navigation, German Aerospace Center, Wessling, 82234 Germany e-mail: florian.moll@dlr.de.

J. Horwath, L. Martin, A. Lozano and D. Diaz are with Vialight Communications GmbH, Gilching, 82205 Germany.

Manuscript received June 06, 2014; revised November 11, 2014, revised March 20, 2015 optical communication terminals can be constructed with remarkably smaller SWaP (Size, Weight, and Power) than RF terminals can offer: first, the antenna size scales down with the wavelength (smaller size and weight) and second, the smaller wavelength enables a lower beam divergence which affects free-space loss and consequently power efficiency. We describe an experimental demonstration of a high-rate airborne optical downlink from a fast flying platform. Fast in this context addresses the aircraft speed being up to 0.7 Mach. Focus was also put on the design of a small and lightweight airborne terminal. The reported aircraft-ground experiment was accomplished within the framework of the DODfast (Demonstration of Optical Data link fast) project which addresses the data dump scenario: downlink of huge amounts of data during a short ground station flyby. For this application, the laser beam propagates through a potential cloud layer. Especially water cloud attenuation in the infrared spectrum reaches extremely high values (as high as 100 up to $600 \mathrm{~dB} / \mathrm{km}$ [1] [2]) and can therefore prohibit line-ofsight for an optical data link. Hybrid solutions (RF/optical) are a viable option here as suggested and investigated in [3] [4] [5]. However, the cloud factor is not a big issue in DODfast-like scenarios. Here, the aircraft is meant to record data during a particular mission nearby or far away from the ground station and store the data temporarily on-board. Once the aircraft seeks line-of-sight to the ground station during the data dump maneuver, downlink is performed. In case of cloud occurrence, the aircraft may descend below the cloud layer, still maintaining line-of-sight to the ground station. Thus, the only remaining obstruction risks are attenuation events in the surface layer like dense fog, haze, and smoke as analyzed in [6] [7] [8]. Because of all its advantages, optical aeronautical communication is in the focus of many research programs all over the world. Successful tracking tests of an aircraft terminal installed in the Boeing 767-200 Airborne Surveillance Testbed are described in [9]. The optical head was mounted inside the aircrafts cabin with laser transmission through an optical window in the fuselage. General design aspects of optical terminals for aircraft and UAVs (Unmanned Aerial Vehicles) are discussed in [10]. A $200 \mathrm{~km}$ link between an aircraft and a ground station on a mountain is described in [11]. Here, the optical head is integrated in a modified Wescam turret attached to the very nose of a BAC 1-11 aircraft. Another turret design is demonstrated with an optical link between an Altair Unmanned Aerial Vehicle and a ground station [12]. An alternative design to the turret version is integration of the actual terminal inside the aircraft body by using an integrated opto-mechanical pointing and tracking assembly for the beam 
steering. Such a system and its demonstration is explained in [13]. Here, a glass dome protects a Coarse Pointing Assembly (CPA) that sticks out of a hole in the lower fuselage of a Dornier Do228. A Coudé path guides the laser beam to an optical bench inside the cabin. A demonstration of a $2.5 \mathrm{~Gb} / \mathrm{s} \mathrm{link}$ over a $25 \mathrm{~km}$ slant path with the terminal installed in the cabin of a Twin Otter aircraft is reported in [14] [15]. Air interface here is an optical window in the side door. Furthermore, a Twin Otter was used in an investigation of channel characteristics for distances between $30 \mathrm{~km}$ to $70 \mathrm{~km}$ from air to ground [16]. In this measurement, a turret installation for the optical terminal was once again applied. The employed laser terminal in our experiments is based on the design described in [13]. However, the CPA and the main terminal unit are miniaturized in a way that a $120 \mathrm{~mm}$ diameter glass dome is the only component exposed to the air stream. The paper is organized as follows: section I gives the motivation for the demonstration, introduces the application, and briefly summarizes the state-ofthe-art of free-space optical communications in the air-ground scenario. Section II outlines the system design, the undertaken link budget assessment, and the scenario of the demonstration. Functionality and operation of the aircraft terminal and the ground station are described in section III and section IV. Section $\mathrm{V}$ shows the demonstration results, i.e., acquisition and tracking performance and link lock. Concluding remarks and outlook are given in section VI.

\section{SYSTEM DESIGN}

\section{A. Concept of demonstration}

Future operational systems will likely use conventional DWDM (Dense Wavelength Division Multiplex) technology to achieve data rates beyond $40 \mathrm{Gbit} / \mathrm{s}$ up to Tbit/s. Since C-band DWDM is state-of-the art in fiber communication technology, laser transmitters, optical amplifiers, and receiver modules are widely available. For a proof-of-concept demonstration, a single wavelength from the DWDM grid in C-band was chosen to demonstrate the feasibility. However, delta development and qualification had to be done in order to fully comply with the applied mil-spec. An atmospheric transmission window exists in $\mathrm{C}$-band and atmospheric extinction and turbulence has considerably lower impact compared to alternative common wavelengths for free-space optical communication such as $850 \mathrm{~nm}$ and $1064 \mathrm{~nm}$. The DODfast system comprises three main units: the airborne laser terminal (Micro Laser Terminal: MLT), the receiver ground station (Transportable Optical Ground Station: TOGS), and the sensor payload. The MLT is installed in the Avionic Demonstrator Tornado (ADT) pod which is attached to the lower fuselage of the Tornado. The demonstration is planned and designed based on deployment of the TOGS as receiver station. It was originally developed to serve for satellite-ground communications and, therefore, the available receiver aperture is rather large for the actual air-ground scenario. This certainly eases the demands on particular system specifications, for instance, divergence of the downlink beam. The TOGS is positioned in the drop zone near the Airbus Defence \& Space Military Air Systems Center at Manching, Germany. The aircraft takes off from

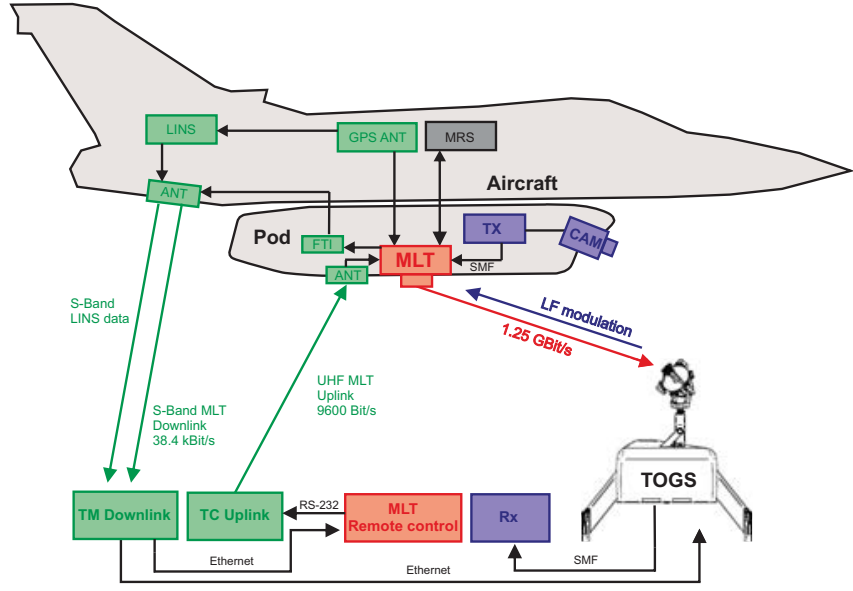

Fig. 1. System overview of the DODfast experiment. The elements of the payload, data source, and sink are labelled purple, the airborne terminal system is red, the TM/TC system is green, and the TOGS is white. FTI denotes part of the Flight Test Instrumentation system in the ADT pod.

the Ingolstadt-Manching airport. The block diagram of the DODfast system is depicted in Figure 1. The function flow is as follows: images or videos are taken by the payload camera and sent to a computer system managing the all onboard high resolution sensors. In the DODfast demonstration, a single camera system is installed and the computer system can be seen as a simple streaming device (Tx). This device generates a $1.25 \mathrm{Gbit} / \mathrm{s}$ UDP data stream interfaced to the MLT with a standard single mode fiber via the on-board fiber optical network. The transmission wavelength is set to a particular DWDM grid channel in C-band. The optical signal is boosted by an EDFA (Erbium Doped Fiber Amplifier) and transmitted towards the TOGS. A mission radiation switch (MRS) is installed in the cockpit to let the pilots enable and disable laser radiation in case of approaching air vehicles or low ground distance. The TOGS receives the signal with a free-space receiver front-end and forwards it to the data sink $(\mathrm{Rx})$ after another e/o-conversion. The MLT is controlled and its performance monitored with a dedicated remote ground station. Therefore, an UHF uplink (antenna installed in the pod) and S-band downlink (antenna installed in the aircraft) are used for the TM/TC (Telemetry/Telecommand) link. Both frequency bands are robust against severe weather conditions and thus provide good reliability.

A second S-band downlink carrying data from the aircraft LINS system provides, amongst other, aircraft GPS data to the TOGS. This is necessary for the applied pointing, acquisition, and tracking scheme: in the first step, open-loop pointing on both sides is performed by using the GPS position of the counter terminal. This procedure aligns the link with an accuracy of a few milliradians. After that, the TOGS beacon lasers hit the aircraft. Tx and Rx beams are separated by optical filtering. With the received beacon signal, the MLT enters the second step, coarse closed-loop optical tracking with its Coarse Tracking Sensor (CTS) and Coarse Pointing Assembly. In the third step, a Fine Tracking Sensor (FTS) and an agile mirror system as Fine Pointing Assembly (FPA) 
TABLE I

SPECIFICATIONS OF THE DODFAST SCENARIO AND COMMUNICATION TERMINALS FOR LINK BUDGET ASSESSMENT.

\begin{tabular}{c||c}
\hline \multicolumn{1}{c||}{ Parameter } & Value \\
\hline \hline Diameter Rx aperture & $600 \mathrm{~mm}$ \\
\hline Diameter Rx aperture obscuration & $158 \mathrm{~mm}$ \\
\hline Field of view TOGS tracking sensor & $4.8 \mathrm{mrad} \times 3.8 \mathrm{mrad}$ \\
\hline Field of view TOGS RFE & $166 \mu \mathrm{rad}$ \\
\hline $\begin{array}{c}\text { Power threshold for stochastic losses } \\
\text { (fading and pointing) }\end{array}$ & $1 \%$ \\
\hline Wavelength downlink (data) & C-band \\
\hline Wavelength uplink (beacon) & C-band \\
\hline Full divergence angle (1/e2) & $2.26 \mathrm{mrad}$ \\
\hline Maximum tolerable MLT tracking error & $200 \mu \mathrm{rad}$ \\
\hline Sensitivity of RFE & $-35 \mathrm{dBm}$ \\
\hline Data rate (IM/DD OOK) & $1.25 \mathrm{Gbit} / \mathrm{s}$ \\
\hline Observer height & $350 \mathrm{~m}$ \\
\hline Atmospheric profile & Mid-latitude summer \\
\hline Cn2-ground value for HV model & $1.7 \cdot 10^{-13} \mathrm{~m}^{-2 / 3}$ \\
\hline
\end{tabular}

enable fast and precise closed-loop optical tracking. A similar algorithm is implemented on the TOGS side. However, only one optical tracking system is applied here. A tracking sensor gives input signals to the control computer that commands the telescope mount with high precision. No additional fine pointing assembly is needed. Eventually, the downlink beam hits the ground station which keeps the signal on the RFE (receiver front-end) diode. Both sides continuously work on acquiring and holding a link lock.

\section{B. Link budget considerations}

Link budgets are analyzed for several flight geometries to assess the feasibility of the project scenario; they are also used during the design of the demonstration to establish possible flight heights, distances, and specific communication terminal specifications. The scenario- and link budget-relevant system specifications are listed in Table 1. The addressed data rate is $1.25 \mathrm{Gbit} / \mathrm{s}$ with intensity modulation, direct detection (IM/DD), and on-off keying (OOK). This modulation scheme, the TOGS telescope size, and the chosen free-space receiver front-end form the starting point for the link budget assessment and scenario design.

The chosen design approach was rather conservative due to expected strong vibrations of the aircraft. This leads to a quite relaxed tolerable tracking error of $200 \mu \mathrm{rad}$ and beam divergence of $2.26 \mathrm{mrad}$ (full 1/e2). The values are both rather large but still guarantee sufficient coupling efficiency for the purpose of the experiment. For a flight height of $3 \mathrm{~km}$ above ground and $20 \mathrm{~km}$ horizontal distance, the link margin ranges from 11.0 to $12.0 \mathrm{~dB}$, and for $40 \mathrm{~km}$ distance from -0.9 to $0.9 \mathrm{~dB}$. The attenuation intervals account for a visibility of 23 and $50 \mathrm{~km}$, respectively. The losses are subdivided into two categories: static and dynamic losses. The former comprise free-space loss, atmospheric attenuation, optics loss, and background light loss. Tx antenna gain, Rx antenna gain, and freespace loss/distance loss are together considered as free-space loss, resulting in 35.2 and $41.1 \mathrm{~dB}$. Atmospheric attenuation is determined using the DLR tool Virtual Lab [17]. Herein, the PFUI (Python Fascode User Interface) tool is a graphical user interface to FASCODE (Fast Atmospheric Signature Code) which incorporates simulation of absorption lines as well as Rayleigh and Mie scattering. Input parameters were observer height, flight heights, distances, a model for atmospheric profile (mid-latitude summer), boundary layer aerosols (rural), and tropospheric visibility. Atmospheric attenuation varies between 3.8 and $4.8 \mathrm{~dB}$ for a $20 \mathrm{~km}$ distance and between 7.4 and $9.2 \mathrm{~dB}$ for a $40 \mathrm{~km}$ distance at a visibility of 23 and $50 \mathrm{~km}$, respectively. Transmission losses in the optics on both sides and receiver sensitivity loss due to background light are also estimated based on practical approximations. The second category includes tracking (Tx and Rx), scintillation, and diode coupling losses. Because of imperfections in the transmitter and receiver tracking systems, pointing and tracking losses occur that have similar influence on the communication system as turbulence-induced intensity fluctuations. The stochastic losses of scintillation and Tx pointing are determined by a signal threshold that causes one percent of the signal to fall below this threshold. Following this approach and the model in [18], the Tx tracking loss is set to $3.2 \mathrm{~dB}$, which is true for an optimum trade-off between beam divergence and jitter. Furthermore, the Rx tracking loss is estimated as $1 \mathrm{~dB}$ by an empirical approach described in [19]. Scintillation loss is calculated using [20] and results in 1.8 and $3.3 \mathrm{~dB}$ for a 20 and $40 \mathrm{~km}$ distance respectively. Aside from intensity scintillation, wave-front distortions are an outcome of index-of-refraction turbulence. These lead to a speckle pattern in the focal plane and a broadening of the long-term focal spot. The mean spot size (Gaussian shape) may overframe the detector resulting in a mean diode coupling loss. However, this loss is small because of the rather large diode diameter of the receiver front-end $(<0.3 \mathrm{~dB})$. The test scenario was developed based on this rather conservative link budget assessment. It demonstrates that data communication can be expected to work for flight heights above $3 \mathrm{~km}$ and horizontal link distances of up to 40 $\mathrm{km}$ - depending on the actual visibility - which matches the foreseen scenario of data dump during flyby.

\section{AIRCRAFT TERMINAL}

A new aircraft terminal was developed for the DODfast downlink demonstration. The basis is the design of the Freespace Experimental Laser Terminal II (FELTII) of DLR which was previously used for testing data downlinks from the Dornier Do228 aircraft [13] [21], airborne channel measurements of atmospheric turbulence [22] and quantum communication [23]. Whereas the FELTII essentially serves as an experimental platform for various kinds of experiments, the MLT exploits the potential of the FELTII design in view of miniaturization, increased agility, and robustness and pushes the development towards a commercial product. The more demanding requirements of the scenario - aircraft speed up to Mach 0.7, severe vibration spectrum of the Tornado attached ADT pod, much higher base motion disturbances, and more restrictive mechanical integration constraints - triggered the new 


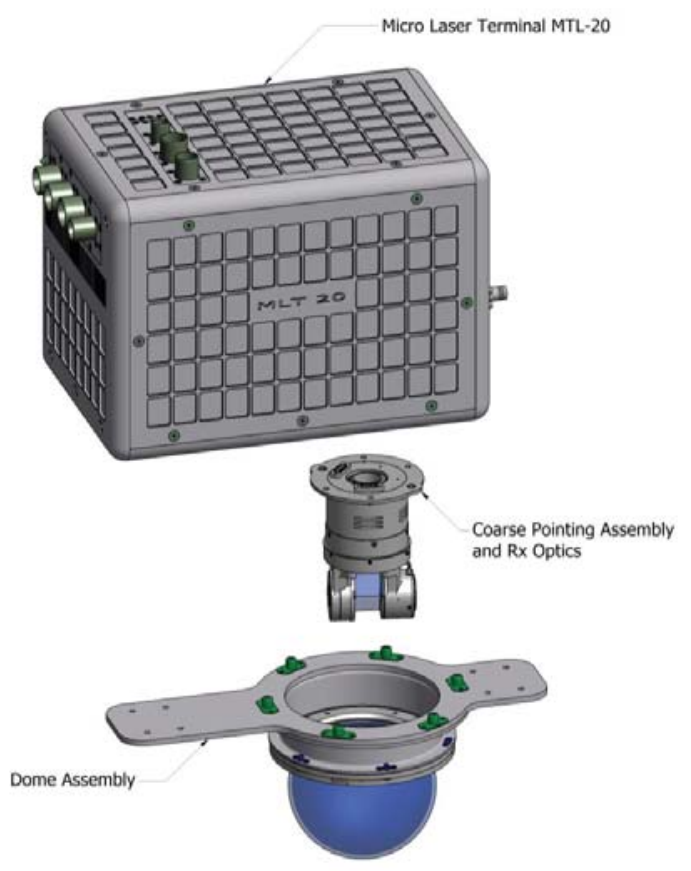

Fig. 2. Main subsystems of the MLT. The box contains all necessary optical, electrical, opto-mechanical, and opto-electrical components. The CPA is attached to the box and the dome assembly to the lower fuselage of the ADT pod.

development. Furthermore, new data interfaces to the TM/TC link, the INS/IMU (Inertial Navigation System/Measurement Unit), and the payload were necessary. The main units of the MLT are shown in Figure 2: the shoebox-sized main housing, the Coarse Pointing Assembly with the receiver optics, and the dome assembly. The Coarse Pointing Assembly is attached to the MLT main body, ducted through a hole, and protected by an optical glass dome that is mounted to the lower fuselage of the ADT pod. The split into these three main elements enables easy and fast installation and gives the possibility of shock-mounting the MLT main body in order to attenuate the vibration amplitudes. This feature was the main driver for the chosen dome size of $120 \mathrm{~mm}$ as this enables very large deflections of the CPA within the dome. The clearance is in the order of several centimeters to accommodate for shocks larger than $40 \mathrm{~g}$. However, due to the ruggedized system design, the chosen beam divergence, and the ability of the active system to compensate broadband vibrations, the idea of using shock mounts to attenuate the vibration spectrum was dropped. A dome shutter to protect from raised debris and dust during the take-off and landing phase is foreseen in the design but has not been implemented for the test phase of the optical terminal.

The opto-mechanical block diagram and the assembled terminal are shown in Figure $3 \mathrm{a}$ and $3 \mathrm{~b}$. Payload data to be transmitted through the optical link is supplied to the MLT via single-mode fiber. An aerospace laser module containing an EDFA provides the necessary tuning option for the mean power level to facilitate the different flight heights, distances, and atmospheric conditions.

After amplification, the signal is fed to the collimation optics. The free-space beam is steered by two different pointing assemblies: The CPA and the FPA. The CPA provides a field of regard for the transmit and receive beams larger than half a hemisphere, while the FPA is a fast electro-mechanical actuator used to compensate higher frequency vibrations. An initial set of pointing angles for the acquisition phase is created based on information about the aircrafts position and attitude supplied by the IMU. On the receiver path, the stabilized optical beam is guided to an Rx/Tx separation system to split the two beams through a subsequent filter (BP). The beam is fed to the CTS and the FTS, which are used as feedback sensors for the optical tracking system. The CTS is used for acquisition, as it provides a wide field of view of several degrees. The FTS provides higher resolution and bandwidth than the CTS, making it suitable for use as the fine pointing systems main feedback sensor. The majority of the above-mentioned components are controlled by the Terminal Control Computer (TCC), which is the MLTs actual brain and takes care of the main tasks, such as operational modes management, data acquisition from external and internal sensors, computation of the pointing angles, and generation of control signals for steering the CPA. For that purpose, inputs from the IMU, the CTS, and the CPA are necessary. Furthermore, it is interfaced to the TM/TC link to receive commands from the ground station. The FPA controller is implemented separately due to the higher control loop bandwidth requirements. It receives data from the FTS, the FPA, and the rate gyros. The flight terminal control software on the TCC is divided in several functional blocks as shown in Figure 4. The terminal core software receives the telemetry information from the partner terminal. Thus, knowing the position and orientation of the terminals, the core software calculates the pointing angles and commands the CPA to point to the ground station accurately enough such that the optical spot is seen on the tracking camera. Then, it commands the CTS to compute the deviation of the spot from the centroid and compensates it. Finally, it commands the FPA controller to further fine-tune the tracking. This way, a beam stabilization of the smaller but faster beam deflections is achieved; these deflections are mainly caused by higher frequency aircraft vibrations and dynamic aberrations due to atmospheric turbulences. The terminal core software also implements the automatic tracking control which generates different control signals depending on the current tracking phase: mutual acquisition phase, coarse tracking phase, fine tracking phase, or re-acquisition phase (in case the link is lost).

\section{RECEIVER GROUND STATION}

DLRs Transportable Optical Ground Station [24] was selected as receiver station for the DODfast demonstration. It was modified and tuned in terms of tracking and pointing accuracy to meet the requirements of the demonstration. The station holds a pneumatically deployable Ritchey-Chretien type aluminum mirror telescope. Once unfolded, it has an optical axis height of around $3.3 \mathrm{~m}$ above ground (Figure 5a). Four mountable supports level the station and compensate surface irregularities. An optical system at the back of the telescope guides the received beam to the tracking camera 


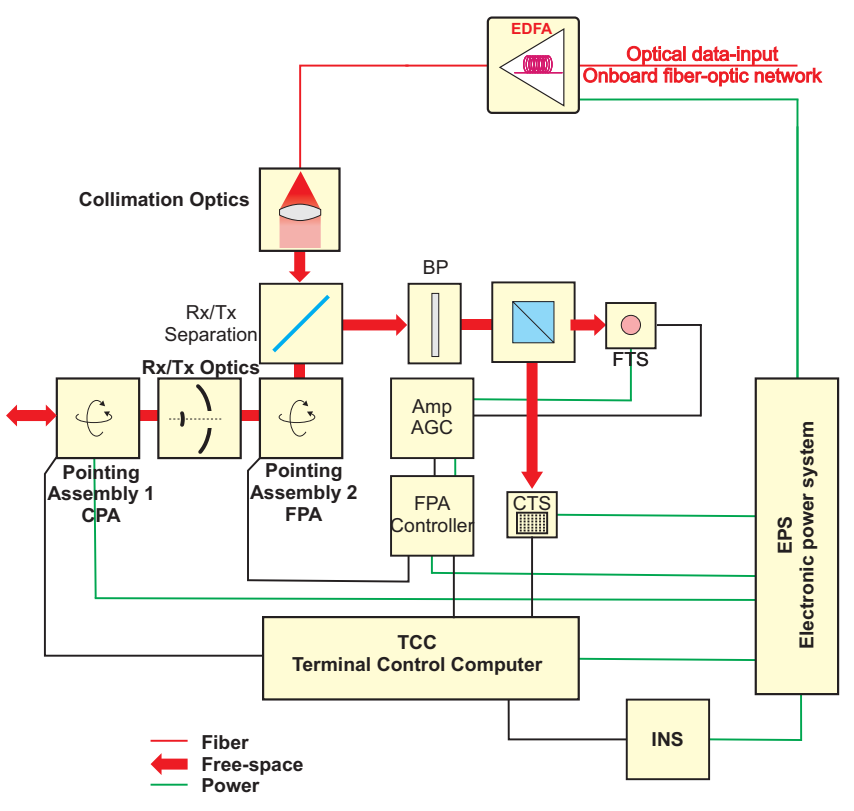

(a) MLT block diagram

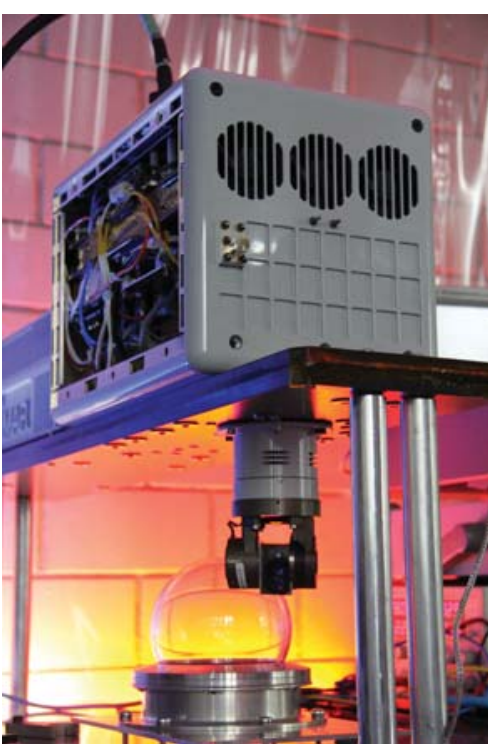

(b) Assembled MLT in laboratory

Fig. 3. MLT System Overview (a). The electrical signal lines are shown in black and optical signals are in red. The power lines are depicted in green. Graph (b) shows an image with the assembled MLT in the laboratory. Source: Vialight Communications

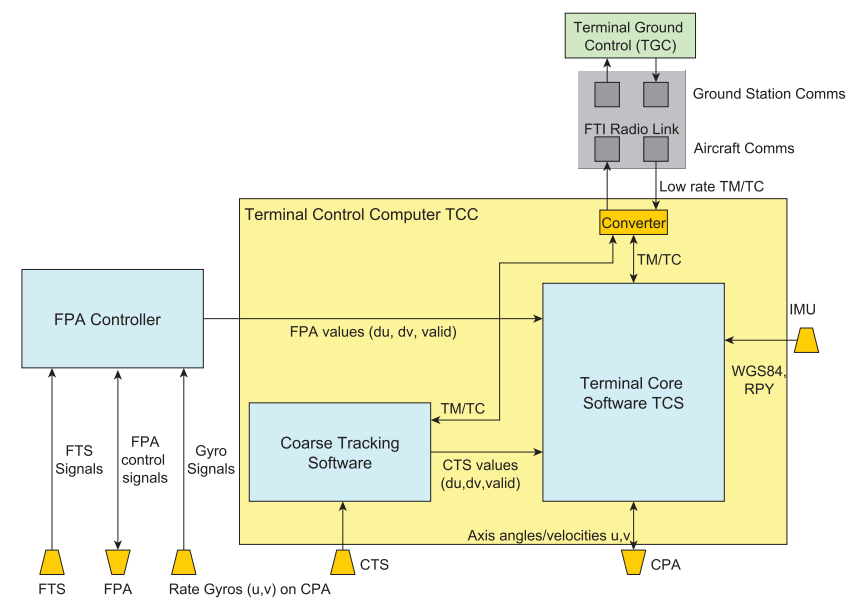

Fig. 4. Overview of the computer systems in the laser terminal with sensor and actuator interfaces: the Fine Pointing Assembly (FPA) controller and the Terminal Control Computer (TCC).

and the free-space RFE as illustrated in Figure 5b. No extra fine pointing assembly is installed and, therefore, tracking performance relies solely on the accuracy of the telescope mount control. To support optical acquisition and tracking on the MLT side, a beacon laser system operating in Cband is applied. Its collimation optics as well as a visible light monitoring camera which is used for cross-checks of the pointing direction are installed aside the telescope. The laser power is boosted by an EDFA to generate the necessary level at the MLT aperture plane. The power can be adjusted to account for different link distance, flight height, and atmospheric extinction.

A movable collimation lens enables adjusting the focus for

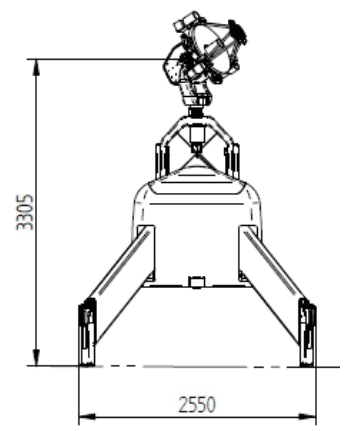

(a) Sketch of TOGS

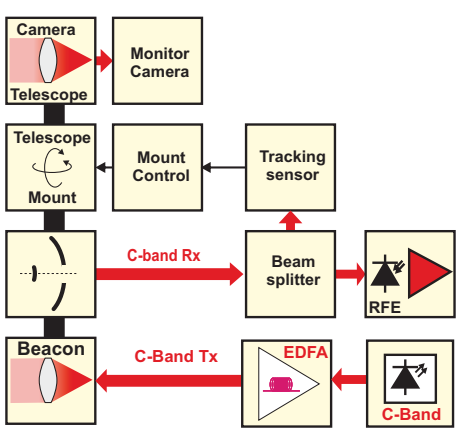

(b) Block diagram
Fig. 5. Technical sketch of unfolded TOGS with dimensions [mm] (a) and block diagram of optical system behind telescope (b). The red arrows mark optical paths, the black arrows electrical connections. The black bar connects items that are attached to the mount.

varying link distances. A beam splitter cube divides the optical power to the tracking camera and the RFE. The pointing, acquisition, and tracking (PAT) process is supported by a single control computer. The block diagram of this process is depicted in Figure 6. Initially, the software calculates the target azimuth and elevation (Az, El) angles needed by the telescope mount to point towards the aircraft terminal by using GPS positions of the TOGS and aircraft. The TOGS continues this open-loop, GPS-based tracking until the signal is seen by the tracking sensor. Eventually, the more precise closed-loop optical tracking takes over to stabilize the beam within the RFEs field of view, thus locking the link. After this acquisition phase, continuous tracking is performed using inputs from the tracking sensor. The downlink communications beam is used as a beacon source and focused on the tracking sensor. Spot positions are related to viewing angles 


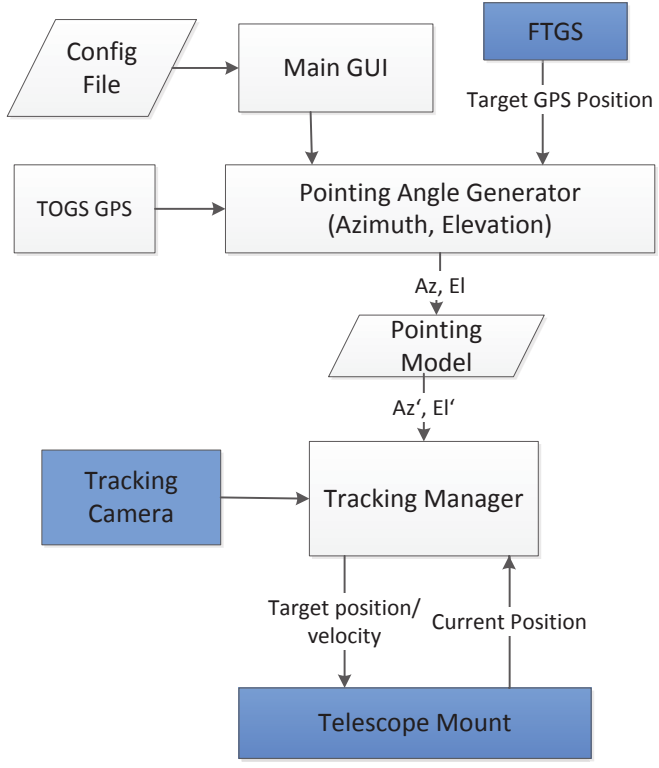

Fig. 6. Block diagram of the TOGS control software.

and respective angular tracking errors. The telescope mount is controlled to minimize these errors. The control loop process runs in near-real-time and continues until the signal is lost and re-acquisition is required. The opto-mechanics of the TOGS may bear various alignment and fabrication errors, such as small offsets in azimuth and elevation direction, nonperpendicularity of the mechanical and optical axes, tilt of azimuth axis towards North, tilt of azimuth axis towards East, and telescope flexure as outlined in [25]. These non-idealities lead to errors in calculation of the azimuth and elevation pointing angles $(\mathrm{Az}, \mathrm{El})$ and may thus delay or even prevent link acquisition. The errors are compensated with a special mount model which is created by a star calibration process similar to that of astronomical telescopes, e.g., [26]. For that matter, the telescope is commanded to track stars that are welldistributed all over the sky hemisphere. Star tracking error measurements are used to calculate the pointing model that realistically describes the mount behavior. This model is then used during the PAT process for calculating corrected target azimuth and elevation angles (Az', El'). A star calibration was performed on site well in advance of the flight campaign to obtain best possible open-loop pointing precision. In bad weather conditions, referencing is done by means of a twoantenna GPS system and particular GPS targets.

\section{EXPERIMENT AND RESULTS}

The flight campaign was conducted in November/December 2013. The Tornado took off from the southern runway of the airport Ingolstadt-Manching. Ground crew for operation of the TOGS, the remote control station of the MLT, the TM/TC links, and experiment operations were on the site. A detailed flight plan was used to most efficiently exploit the limited air time. The single flights were subdivided into individual runs,

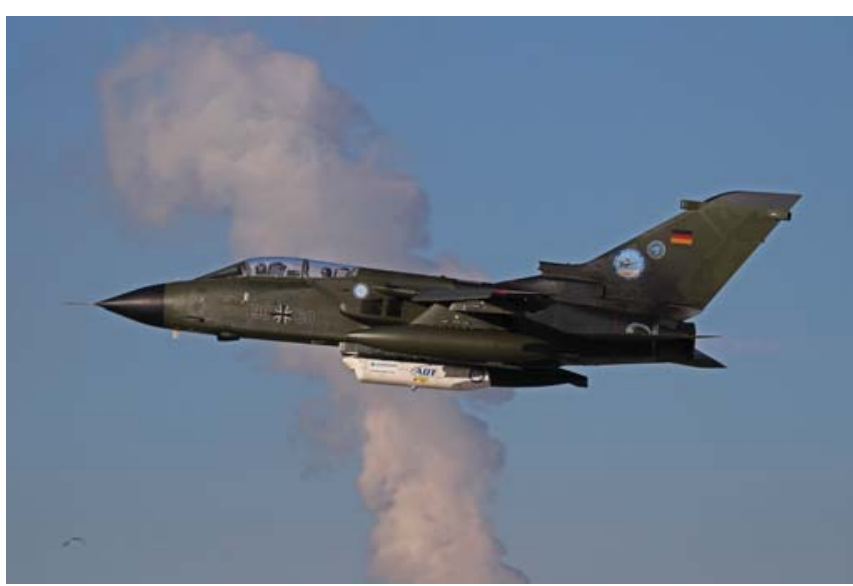

(a) Tornado aircraft

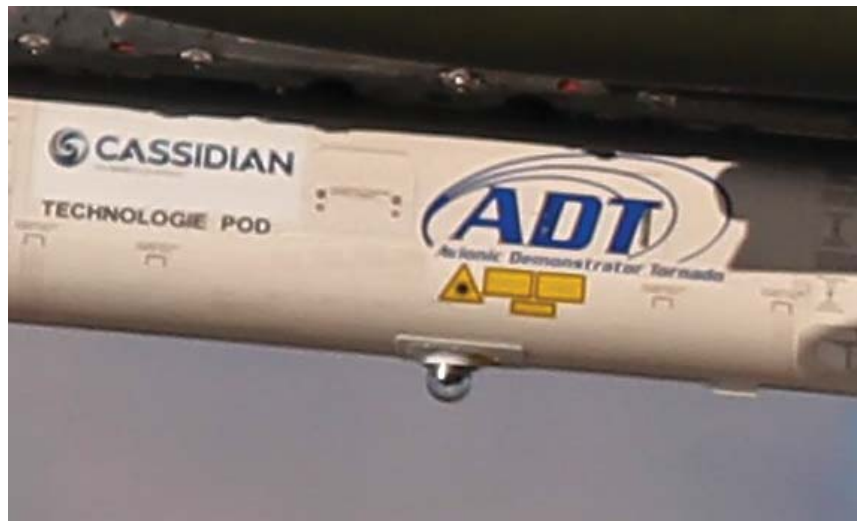

(b) Zoom of MLT dome

Fig. 7. Illustration of DODfast demonstration: Tornado with attached ADT pod (a) and close-up of pod revealing the MLT glass dome (b). Source: Josef Gietl/Airbus DS.

each with particular flight path geometry (circular, tangential, radial), different azimuth directions towards the ground station, flight speed and height. This enabled the crew to react to occurring cloud fields during the experiment run time and test various flight conditions. Two flights are reported here. The weather situation during the first flight was overcast, hindering link acquisition during the planned flight paths. However, special low approaches towards the airport allowed the aircraft to dive below the cloud layer for very short times (several seconds). This was sufficient to receive a beacon signal, step into tracking mode, lock the link, and transmit a short video sequence. During the second flight weather conditions were better (with almost clear sky but some aerosol content ${ }^{1}$ ), providing good preconditions for performance measurements. Several of the planned flight paths could be conducted. Figure 7a shows an image of the aircraft with attached ADT pod during the second flight. Figure $7 \mathrm{~b}$ shows a zoom revealing the small glass dome.

The ground station setup is shown in Figure 8. The TOGS is unfolded and partly sheltered by the transport vehicle. The receiver antenna for the telemetry link is located on the rooftop

${ }^{1}$ Pilots reported hazy conditions in the visible range for line of sight $>10$ $\mathrm{km}$ 


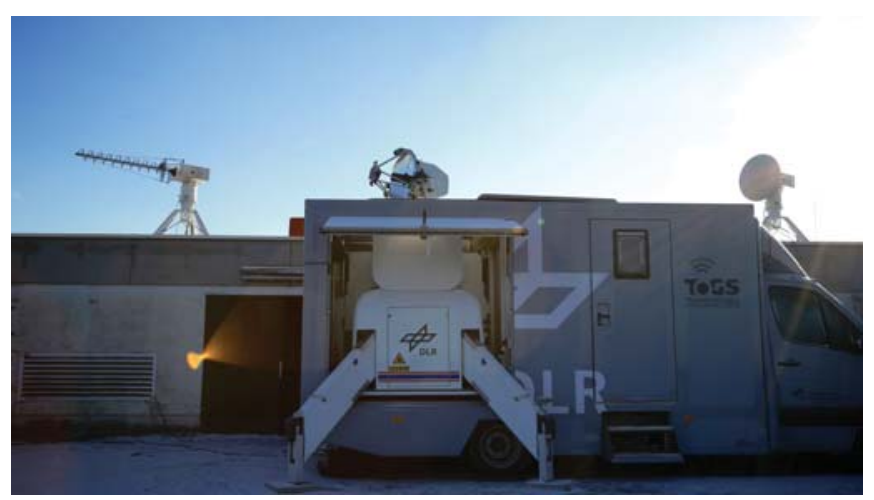

Fig. 8. DODfast demonstration ground setup with TOGS and TM/TC antennas at drop zone. Source: DLR

of the house behind the TOGS van on the right-hand side, the one for the telecommand on the left.

The path map of flight \#02 is shown in Figure 9. The flight was organized in 13 separated runs (indicated as black lines). The red coloring of the runs lines depicts the status TOGS tracking active but no signal on RFE, meaning MLT acquired the link and TOGS is in the acquisition phase. The green lines indicate successful link acquisition, which is defined by exceeding a RSSI (Received Signal Strength Indicator) threshold of the RFE. A successful link acquisition requires all involved algorithms and subsystems, such as the TOGS/MLT pointing scheme, the TOGS/MLT tracking, power adjustment on both sides, and TM/TC infrastructure, to be fully functioning. The link was locked most of the time. The total experiment time was $5707 \mathrm{~s}$, which is the sum of all run durations. The MLT CTS has the highest fractional link time ( $88 \%)$, since it is the first sensor in the acquisition process to receive a signal. The second sensor in the chain is the MLT FTS with a similar link time (84\%). When both MLT sensors have a valid signal, the MLT is in tracking mode and the beam is expected to hit the TOGS, i.e., its acquisition and tracking sensor. Thus, the TOGS achieved $74 \%$ link time. Ultimately, a data signal lock was achieved during $52 \%$ of the experiment time (defined by the RSSI threshold). Some issues that prevented the system from having $100 \%$ link time could be identified. The reasons are twofold: first, line of sight was occasionally blocked by cloud fields and the aircraft bank angle during some maneuvers. Second, a few technical issues were present. Since no fully operational automated power control was implemented, incident irradiance on the aperture was sometimes very high which caused the RFE to run into saturation. Under this condition, the RFE output signal level drops and the communication link is lost. Similar issues occur during the beacon uplink. A saturation of the MLT FTS in the acquisition phase may cause the signal processing algorithm to label a detected spot as invalid and therefore lead to an acquisition delay. On the other hand, signal power on the MLT and TOGS side was occasionally too low and had to be increased. Furthermore, the signal delay in the transmission of GPS positions caused a systematic pointing offset on the TOGS side. A lag compensation was implemented, however,

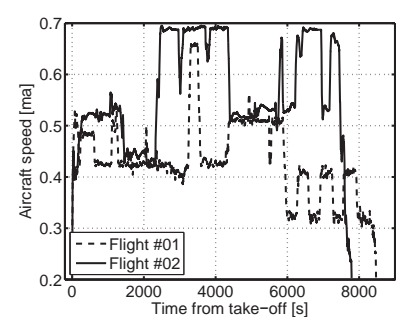

(a) Aircraft speed

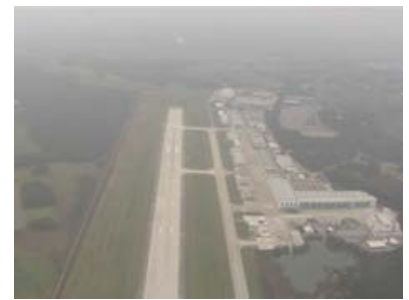

(b) Sensor image
Fig. 10. Flight speed of aircraft during the DODfast test flights (a). The targeted flight speed was reached several times. The right image (b) shows a snapshot of the payload sensor. Source: Airbus DS

the real signal delay was not known a priori and needed to be adjusted during runtime. Also, defocus of the TOGS tracking sensors due to changing distances was observed and had to be compensated manually.

The speed of the aircraft during the experiment is shown in Figure 10a. The aircraft reached maximum speed of Mach 0.7 (during several runs in flight \#02). Faster flight speeds would also have been possible from the perspective of the laser link. However, airworthiness certification issues prohibited even higher speeds. One of the first transmitted images is shown in Figure 10b. The scene shows the southern runway of airport Ingolstadt-Manching and was taken during one of the low approaches during flight \#01. Eventually, the optical link could be established during flight \#01 and \#02. The maximum distance of active optical closed-loop tracking was $79 \mathrm{~km}$. Data communication, i.e., video transmission, could be achieved over up to $50 \mathrm{~km}$ distance. The link budget predicts a maximum distance of $40 \mathrm{~km}$. Due to varying atmospheric conditions, this distance can be longer in better conditions or shorter in worse conditions. The longer tracking distances are due to a higher robustness of the tracking link. In the current setup, signal fluctuations affect the communication link stronger than the tracking link. The conservative link budget was ultimately outperformed in flight \#02 with way longer distances than expected. Even longer distances can be reached by reduction of the used beam divergences, which is technologically feasible with little effort. However, the applied divergence here fully satisfied the scenario constraints.

The tracking error of the MLT and the TOGS is shown in Figure 11a, 11b, 11c and 11d. Graphs 11a and 11b contain measurements for flight \#02, run \#12 (350-400 s). The flight geometry was a tangential flyby. Graphs $11 \mathrm{c}$ and $11 \mathrm{~d}$ show the measurements for run \#13 (150-200 s). Here, the flight geometry was a radial inbound maneuver. The ground speed for run \#12 was $\sim 240 \mathrm{~m} / \mathrm{s}$, flight height $\sim 2750 \mathrm{~m}$, and distance between 55 and $68 \mathrm{~km}$. A ground speed of $240 \mathrm{~m} / \mathrm{s}$ converts into Mach 0.7 at the aircraft altitude. The ground speed for run \#13 was $\sim 160 \mathrm{~m} / \mathrm{s}$, flight height $\sim 2850 \mathrm{~m}$, and distance between 48 and $58 \mathrm{~km}$. For evaluation of the MLT tracking performance, the readings from the fine tracking sensor are used. The instantaneous MLT tracking error stayed well below $100 \mu \mathrm{rad}$ for run \#12 (Figure 11a) and even below $60 \mu \mathrm{rad}$ for run \#13 (Figure 11c) with rms values of 40 and $20 \mu \mathrm{rad}$, respectively. Therefore, tracking performance is much better 


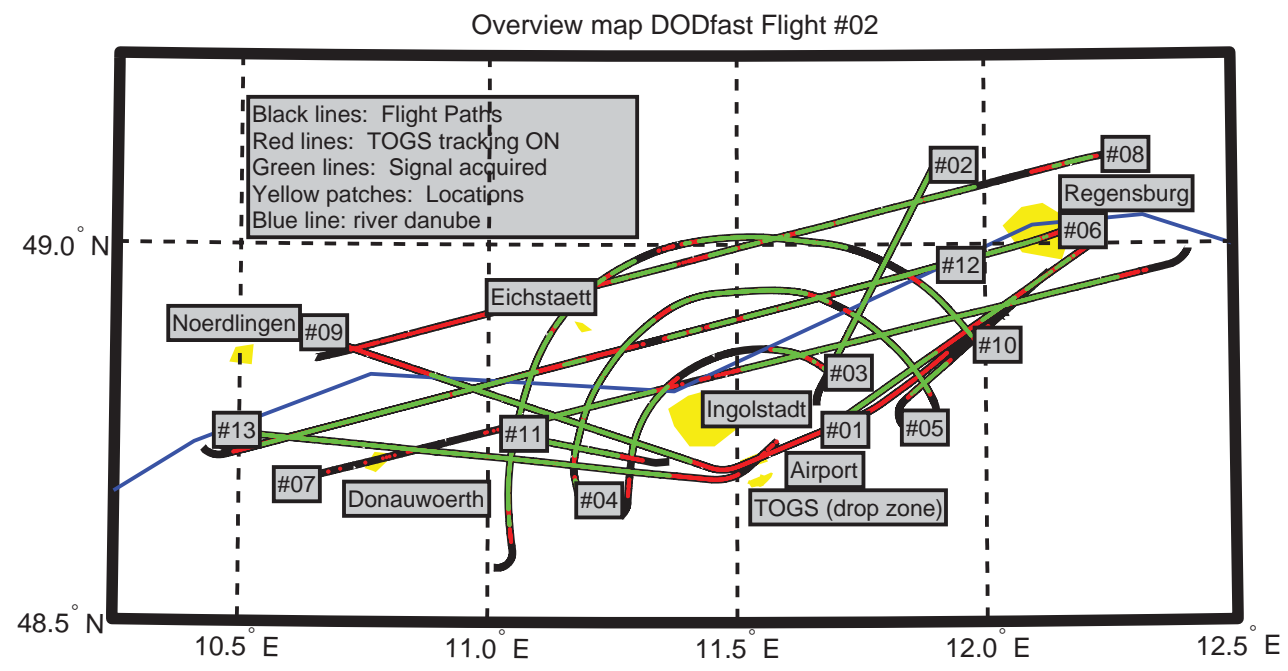

Fig. 9. Overview of flight paths during flight \#02. The lines are color-coded to illustrate times of acquisition (TOGS tracking ON) and signal lock (signal acquired). The yellow patches mark the surrounding cities and the airport zone. The blue line denotes the river Danube.

than required by the link budget $(200 \mu \mathrm{rad}$ std). However, without the severe vibration levels at the MLT mounting points, which even showed some resonances, this number would be even smaller around some factors. No considerable outages could be observed and stable tracking was obtained over the whole run. The tracking performance on the TOGS side showed similar stability. The output of the spot detection algorithm is used to estimate the TOGS tracking error. The instantaneous tracking error was well below $40 \mu \mathrm{rad}$ for run \#12 (Figure 11b) and below $100 \mu \mathrm{rad}$ for run \#13 (Figure 11d). The rms error was $20 \mu \mathrm{rad}$ and $45 \mu \mathrm{rad}$, respectively.

The RSSI is determined using an envelope detector with the electrical analog output of the RFE. This RSSI gives the ultimate information on link lock times and stability. Figure 12 a shows the RSSI over time for run \#12 (same time interval as in Figure 11a and 11b). Figure $12 \mathrm{~b}$ shows the RSSI over time for run \#13 (same time interval as in Figure 11c and 11d). The dashed line denotes the empirically set threshold to define link acquisition yes/no.

In both runs, the RSSI is high enough for feasible data reception and shows similar stability. However, the analysis of tracking performance in Figure 11a, 11b, 11c and 11d shows a different trend for the MLT and for the TOGS performance. For the MLT, the tracking error is higher in run \#12 and lower in run \#13. This comes from the higher aircraft speed in run \#12 which corresponds to higher amplitudes of vibrations. On top of this, the roll of the aircraft has the strongest impact during a tangential flight path, thus providing an even greater challenge to the tracking system. The tracking error in run \#13 is lower. On the TOGS side, the situation is vice versa. The higher aircraft speed does not challenge the TOGS tracking system since the downlink beam is still very stable (low MLT tracking error). However, in the case of a radial inbound flight, the angular aircraft movement seen by the TOGS is very low. Since the tracking control loop is optimized for moderate and

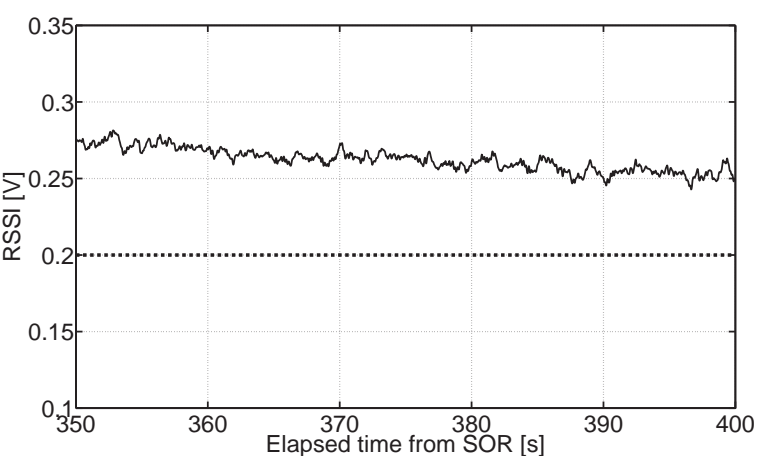

(a) RSSI run 12

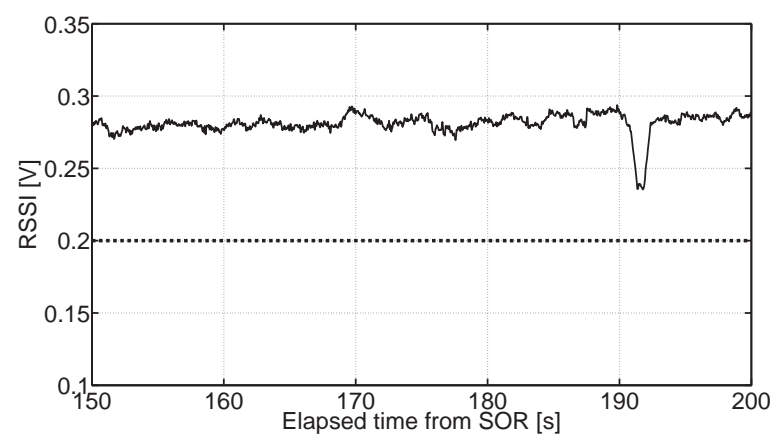

(b) RSSI run 13

Fig. 12. RSSI of received signal during flight \#02 run \#12 (a) and run \#13 (b). The black solid line denotes the RSSI [V], The dashed line marks the valid signal threshold. SOR: Start Of Run.

high velocities, the tracking error is higher here. Ultimately, the TOGS tracking error in Figure $11 \mathrm{~b}$ is still low enough to keep the signal spot on the RFE diode within its $166 \mu \mathrm{rad}$ field of view. 


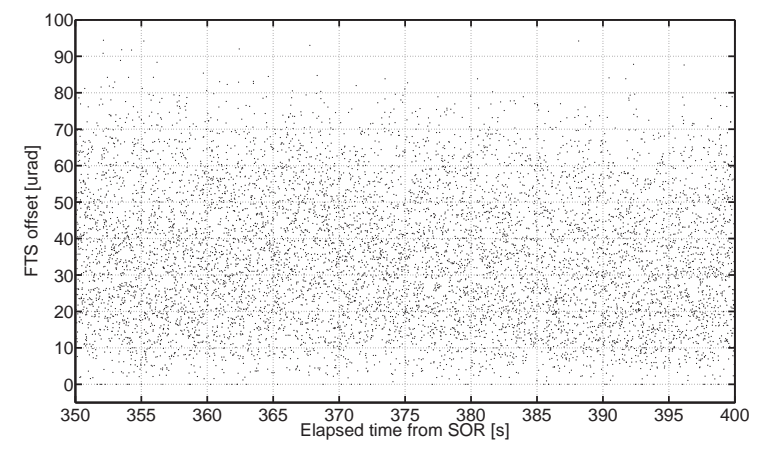

(a) MLT run 12

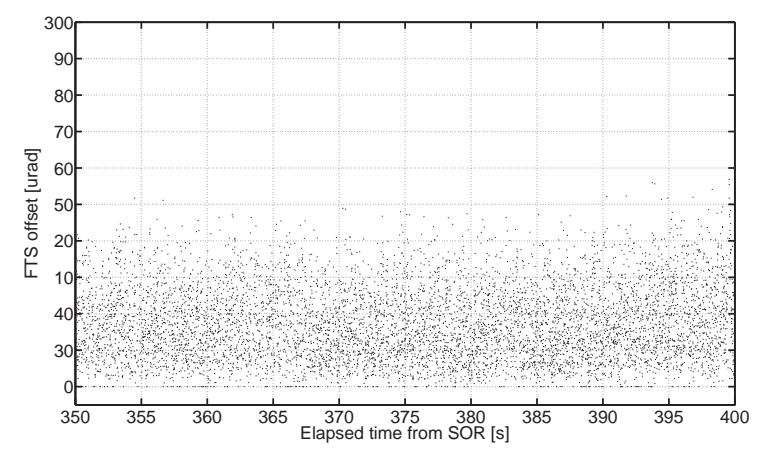

(c) MLT run 13

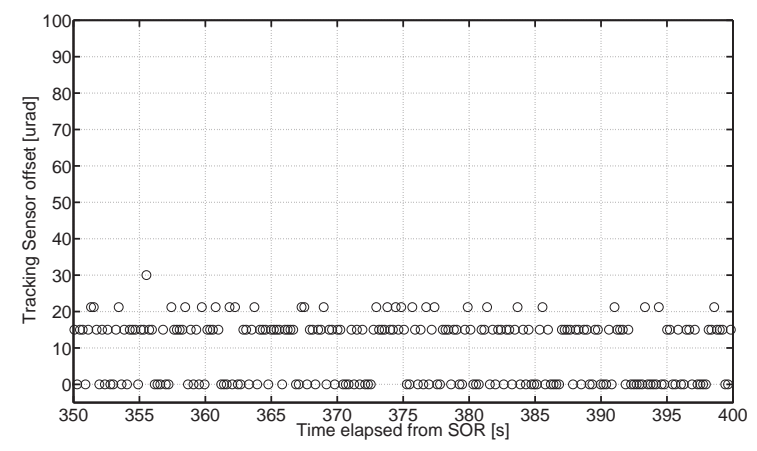

(b) TOGS run 12

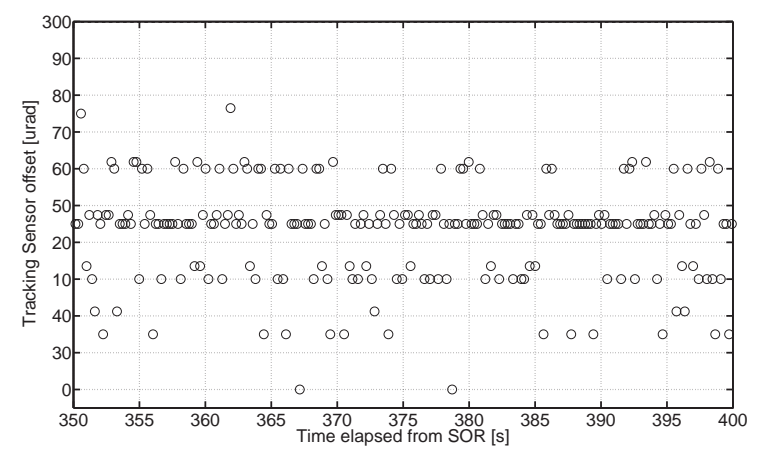

(d) TOGS run 13

Fig. 11. Excerpt of tracking error measurement from flight \#02, run \#12 for MLT (a) and TOGS (b) and run \#13 for MLT (c) and TOGS tracking sensor (d). SOR: Start Of Run.

\section{SUMMARY AND CONCLUSION}

We reported on the demonstration of a high-rate optical downlink from a fast flying platform to a ground station. To the authors knowledge, this was the first demonstration of an optical link from a fast flying jet fighter aircraft to a ground station (with a speed of up to Mach 0.7). Tracking distances were as high as $79 \mathrm{~km}$; data communication could be achieved up to $50 \mathrm{~km}$. The aircraft terminals and ground stations specifications were set with an initial link budget estimation. In the demonstration, the flown system greatly outperformed the previously assessed link budget and link distances. The maturity of aeronautical free-space laser communication within the context of worldwide ongoing research and development was demonstrated. Both aircraft and ground terminal systems fully satisfied the demanded performance and, on top of that, the degree of integration on the aircraft side is unreached to date. This is also true for the transportable ground station. In both systems, we identified several subsystems that still affect acquisition and tracking performance and should be optimized. Amongst these are automatic power control on the aircraft and ground station side, automated focus control, delay in aircraft position transmission, and gain scheduling of control loop parameters. In addition, the MLT dome size can be halved because of the possibility of dropping the passive shock mounts. Furthermore, the used system already offers lots of potential to lower the beam divergence while still keeping the tracking error induced signal fluctuations low. Thus, these tracking systems bear high potential and improvements will be the focus of future experiments when coupling efficiency must be increased due to higher data rates or smaller receiver antennas. Both will be encountered in case of ground-air uplink communication or for optical inter-aircraft links. Especially the later scenario is of growing interest for implementation in passenger aircraft to satisfy the need of future aeronautical backbone networks [27].

\section{ACKNOWLEDGMENT}

The DODfast project is a collaboration of the German Aerospace Center (DLR) Institute of Communications and Navigation, Airbus Defence \& Space, and ViaLight Communications. The flight campaign was organized and conducted together with the Bundeswehr Technical and Airworthiness Center for Aircraft (WTD 61) in Manching. The authors would like to thank everyone who was involved in this project and the conducted flight campaign. Special thanks go to Mr. Wolfgang Mitzkus as DODfast project manager and also Mr. Marco Bobbio and DLR SHT who greatly contributed to the projects success. We would also like to thank all members of Airbus Defence \& Space who were involved in the TM/TC link setup, MLT integration, and the flight campaign. Finally, we would like to give special thanks to the team of the Bundeswehr Technical and Airworthiness Center for Aircraft (WTD 61) in 
Manching who supplied the aircraft and provided tremendous support in all phases of the demonstration.

\section{REFERENCES}

[1] F. Moll and M. Knapek, "Wavelength selection criteria and link availability due to cloud coverage statistics and attenuation affecting satellite, aerial, and downlink scenarios," in Proceedings of SPIE, vol. 6709, 2007, pp. 670916-1 - 670916-12.

[2] J. H. Churnside and K. Shaik, "Atmospheric propagation issues relevant to optical communications," NOAA, Boulder, Colorado, NOAA Technical Memorandum ERL WPL-159, 1989.

[3] H. Wu and M. Kavehrad, "Availability evaluation of ground-to-air hybrid fso/rf links," International Journal of Wireless Information Networks, vol. 14, no. 1, pp. 33-45, 2007.

[4] B. L. B. Stotts, L. C. A. P. C. Cherry, J. J. Foshee, P. J. Kolodzy, W. K. McIntire, R. L. Phillips, H. A. Pike, B. Stadler, and D. W Young, "Hybrid optical rf airborne communications," in Proceedings of the IEEE, vol. 97, no. 6, 2009, pp. 1109-1127.

[5] Z. C. Bagley, D. H. Hughes, J. C. Juarez, P. Kolodzy, T. Martin, M. Northcott, H. A. Pike, N. D. Plasson, B. Stadler, L. B. Stotts, and D. W. Young, "Hybrid optical radio frequency airborne communications," Optical Engineering, vol. 51, no. 5, pp. 055006-1-055006-25, 2012.

[6] M. Grabner and V. Kvicera, "The wavelength dependent model of extinction in fog and haze for free space optical communication," Optics Express, vol. 19, no. 4, pp. 3379-3386, 2011.

[7] M. A. Naboulsi, H. Sizun, and F. de Fornel, "Fog attenuation prediction for optical and infrared waves," Optical Engineering, vol. 43, no. 2, pp. 319-329, 2004

[8] M. Ijaz, Z. Ghassemlooy, J. Pesek, O. Fiser, H. L. Minh, and E. Bentley, "Modeling of fog and smoke attenuation in free space optical communications link under controlled laboratory conditions," Journal of Lightwave Technology, vol. 31, no. 11, pp. 1720-1726, 2013.

[9] V. J. Chan and R. L. Arnold, "Results of one gbps aircraft-to-ground lasercom validation demonstration," in Proc. SPIE 2990, Free-Space Laser Communication Technologies IX, vol. 2990, 1997, pp. 52-59.

[10] H. Henniger and D. Giggenbach, "Avionic optical links for high datarate communicaations," in Proceedings of 25th Congress of International Council of the Aeronautical Sciences, 2006.

[11] L. B. Stotts, "Optical communications in atmospheric turbulence," in Proc. of SPIE, Free-Space Laser Communications IX, vol. 7464, 2009.

[12] G. G. Ortiz, S. Lee, S. P. Monacos, M. W. Wright, and A. Biswas, "Design and development of a robust atp subsystem for the altair uav-toground lasercomm 2.5-gbps demonstration," in Proc. SPIE 4975 (FreeSpace Laser Communication Technologies XV), vol. 4975, 2003.

[13] J. Horwath and C. Fuchs, "Aircraft to ground unidirectional lasercommunications terminal for high-resolution sensors," in Proc. SPIE Free-Space Laser Communication Technologies XXI, vol. 7199, 2009.

[14] F. G. Walther, S. Michael, R. R. Parenti, and J. A. Taylor, "Air-to-ground lasercom system demonstration design overview and results summary," in Proc. of SPIE (Free-Space Laser Communications X), vol. 7814, 2010 pp. 78 140Y-78 140Y-9.

[15] F. G. Walther, G. A. Nowak, S. Michael, R. Parenti, J. Roth, J. Taylor, W. Wilcox, R. Murphy, J. Greco, J. Peters, T. Williams, S. Henion, R. Magliocco, T. Miller, and A. Volpicelli, "Air-to-ground lasercom system demonstration," in Proceedings of the Military Communications Conference, 2010, pp. 2295-2301.

[16] L. C. Andrews, R. L. Phillips, R. Crabbs, T. Leclerc, and P. Sauer, "Final report: channel characterization for free-space optical communications,' UCF DARPA, Tech. Rep., 2012.

[17] T. Ernst, T. Rother, F. Schreier, J. Wauer, and W. Balzer, "Dlr's virtuallab: Scientific software just a mouse click away," Computing in Science and Engineering, vol. 5, no. 1, pp. 70-79, 2003.

[18] K. Kiasaleh, "On the probability density function of signal intensity in free-space optical communications systems impaired by pointing jitter and turbulence," Opt. Eng., vol. 33, no. 11, pp. 3748-3757, 1994.

[19] F. David, D. Giggenbach, H. Henniger, J. Horwath, R. Landrock, , and N. Perlot, "Design considerations for optical inter-hap links," in Proceedings of the 22nd ICSSC, 2004.

[20] D. Giggenbach and H. Henniger, "Fading-loss assessment in atmospheric freespace optical communication links with on-off keying," Opt. Eng., vol. 47 , no. 4 , p. $046001,2008$.

[21] C. Schmidt, J. Horwath, A. Shrestha, F. Moll, M. Brechtelsbauer, and C. Fuchs. (2013) High-speed, high-volume optical communication for aircraft.
[22] K. Shortt, D. Giggenbach, R. Mata-Calvo, F. Moll, C. Fuchs, C. Schmidt, J. Horwath, J. Yeh, V. Selvaraj, and R. Banerjee, "Channel characterization for air-to-ground free-space optical communication links," in Proc. SPIE 8971 (Free-Space Laser Communication and Atmospheric Propagation XXVI), vol. 8971, 2014.

[23] S. Nauerth, F. Moll, M. Rau, C. Fuchs, J. Horwath, S. Frick, and H. Weinfurter, "Air-to-ground quantum communication," Nature Photonics, vol. 7, p. 382386, 2013.

[24] C. Fuchs, M. Brechtelsbauer, J. Horwath, A. Shrestha, F. Moll, D. Giggenbach, and C. Schmidt, "Dlrs transportable optical ground station," in Proc. of Applications of Lasers for Sensing and Free Space Communications (OSA Technical Digest), 2013.

[25] A. Shrestha and M. Brechtelsbauer, "Transportable optical ground station for high-speed free-space laser communication," in Proc. SPIE 8517 (Laser Communication and Propagation through the Atmosphere and Oceans), vol. 8517, 2012.

[26] A. Hempelmann, J. G. Perez, J. Schmitt, and H. Hagen, "The hamburg robotic telescope: a test report," in Proc. 13th Cool Stars Workshop, 2004.

[27] K.-D. Bchter, A. Reinhold, G. Stenz, and A. Sizmann, "Drivers and elements of future airborne communication networks," in Proceedings of Deutsche Gesellschaft fr Luft- und Raumfahrt - Lilienthal-Oberth e.V., 2012.

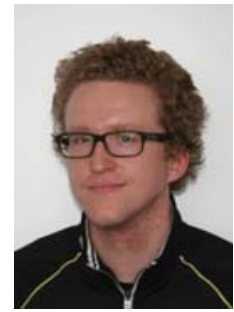

Florian Moll received his Dipl. Ing $(\mathrm{FH})$ in electrical engineering from the Jena University of Applied Sciences in 2006 and a M.Sc. degree in electrical engineering from the Technische Universitae Muenchen (TUM) in 2009. He has been a member of the German Aerospace Centre (DLR), Institute of Communications and Navigation since 2009 and is member of the research group Optical Communications Systems. Here, he is in charge of the Optical Ground Station Oberpfaffenhofen. His main research interests are atmospheric turbulence and optical communications in LEO and aeronautical scenarios. He was DLR project leader for the DODfast demonstration.

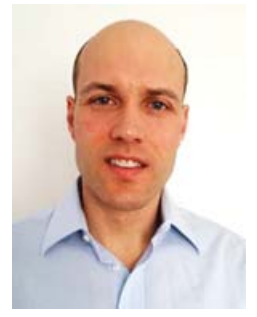

Joachim Horwath received the Dipl.-Ing degree (M.S.) from the Technical University Graz in 2001 In 2002 he joined the German Aerospace Center (DLR) as a research staff member on free-space optical communications systems. He studied atmospheric effects on coherent and incoherent FSO systems by means of experiments and numerical simulation. He did lead several national and international projects dealing with optical communicationsterminal development for remote sensing and telecom. missions flown on different aeronautical platforms. In 2009 he co-founded ViaLight Communications (VLC). Since then he is the VLC lead engineer for all system developments. 


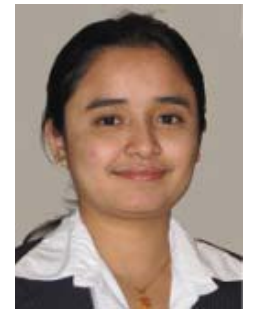

Amita Shrestha is a researcher at German Aerospace Center, DLR. She graduated with her Masters degree in Communication Systems and Electronics from Jacobs University, Bremen in 2007. She completed her undergraduate studies in Electronics Engineering and Communications from Kathmandu University, Nepal in 2006 and worked as a faculty member in Kathmandu University until 2007. Since 2010, she has been working in the Optical Communication Group in the Institute of Communications and Navigation at DLR. Her professional experience and interests are in the area of digital communications, signal processing and software development. She is involved in the development of real-time tracking software for the institute's optical ground station, and in several other projects related to the development of high speed laser Ethernet transceivers.

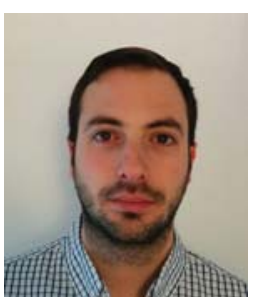

Alberto M. Lozano Souto specialized in Software Engineering at the Universidad de Sevilla (Spain). $\mathrm{He}$ began his career as a Junior Programmer at Infodesa (Sevilla), where he contributed in the project which merged Caja San Fernando and El Monte banks. Before becoming part of Vialight Communications in 2012, he worked for Abance S.L. (Cádiz) where he worked as a Software Engineer developing the software for the new series of Frigates F-105 for the Spanish Navy. Currently he works as Software Vialight Communications terminals.

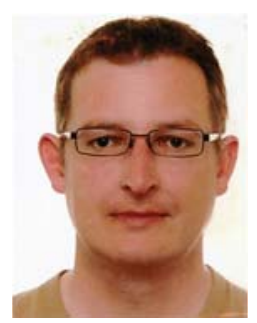

Martin Brechtelsbauer received his Dipl.-Ing and M.Sc. degrees from Munich University of Applied Sciences in 2005 and 2009 respectively. In 2005, he joined DLR as a student. He has been working on different projects involving free-space optical communications. His main field of activity is the development of electronic components for laser communications. He has participated in various measurement campaigns demonstrating optical downlinks from aircraft and satellites. Since 2009. he has led the TOGS (Transportable Optical Ground Station) project, which engages in the development of a transportable optical ground station.

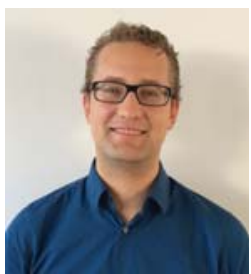

Christian Fuchs graduated in Telecommunications / Electronics from the University of Applied Sciences, Mannheim, Germany in 2006. Since then he has been with DLRs Institute of Communications and Navigation and coordinated several national and international research projects regarding free-space optical communications in aeronautical- and spaceenvironments. Since 2011, he has been leading the Optical Communication Systems Group. His research interests include optical communication sysas well as for GEO-feeder links.

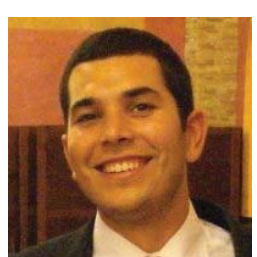

Luis A. Martín Navajas received the B.Sc and M.Sc degrees in telecommunications engineering from the University of Málaga, Spain. He is a system engineer and works since 2013 on the development of free-space optical communication terminals at Vialight Communications $\mathrm{GmbH}$ in Gilching, Germany. He develops the optical tracking system for free-space optics applications. He has worked as a student at the German Aerospace Center (DLR) in Oberpfaffenhofen in 2012 in the area of multisensor fusion at the department of communication and navigation. He has studied one year as an exchange student at the Technical University of Graz, Austria.

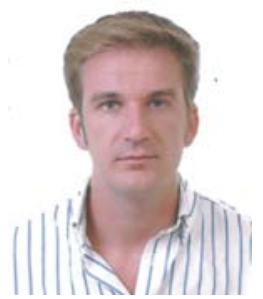

Dionisio Daz Gonzlez specialized in Signal Processing and Radio Communication at the Universidad de Sevilla (Spain). He complemented his studies in Telecommunications Engineering at the Institut Supérieur d'Électronique de Paris (France) and at the Universidad Carlos III de Madrid (Spain). He began his career as a student collaborator in Paris contributing to the project Citypulse in the field of Urban Games for the company Xilabs. After a traineeship at Vodafone Espaa, he joined Triagnosys $\mathrm{GmbH}$ in Wessling (Germany) working on the optimization of Mobile Ipv6 for Satellite Link in an air traffic management network test bed. Before becoming part of Vialight Communications in 2011, he completed his research period in the German Aerospace Center (Gilching, Germany) focusing on the integration of a fine-tracking system in a test bed for adaptive optics. Currently he is developing laser communication terminals for aerial applications and inter-platform connections, expanding his range of tasks as Project Manager, System Engineer and Opto-mechanical Designer. 